\title{
A COMPOSIÇÃO RELATIVA DE ATRIBUTOS APLICADA EM ESCALAS DE DESEMPENHO ACADÊMICO
}

\author{
Luiz Octávio Gavião \\ Universidade Federal Fluminense (UFF) \\ luiz_gaviao@id.uff.br \\ Gilson Brito Alves Lima \\ Universidade Federal Fluminense (UFF) \\ glima@id.uff.br \\ Annibal Parracho Sant'Anna \\ Universidade Federal Fluminense (UFF) \\ annibal.parracho@gmail.com
}

\begin{abstract}
Resumo
Sistemas de classificação auxiliam organizações e instituições nos diversos processos de admissão, seleção e promoção do pessoal. No caso específico da profissão militar, os sistemas de classificação também definem a hierarquia nas turmas de Oficiais e Praças e, por vezes, a prioridade de escolha de futuras comissões, com implicações profissionais, familiares e financeiras. As notas finais utilizadas para a classificação, obtidas nas diversas disciplinas de cursos e concursos, podem ser melhor exploradas sob o aspecto do mérito relativo ou comparado. A finalidade desse artigo é apresentar um método, denominado "Composição Relativa de Atributos", capaz de valorizar o mérito de uma determinada nota, ao considerar sua posição relativa à distribuição das demais notas da mesma disciplina. $\mathrm{O}$ método proposto distribui as notas por gráficos de box plot e associa os intervalos do gráfico a clusters, convertendo as notas absolutas em notas relativas por modelagem fuzzy. O método foi aplicado em uma base de dados hipotética, composta por notas de 40 alunos em dez disciplinas, para distinguir o mérito dos seis primeiros classificados, que obtiveram médias aritméticas idênticas ao término de um período letivo. Os resultados do estudo de caso demonstraram que a distinção do mérito foi eficaz.
\end{abstract}

Palavras-Chaves: Classificação fuzzy; avaliação de desempenho; mérito relativo; box plot.

\begin{abstract}
Grading systems assist organizations and institutions in the diverse admission, election and promotion processes. In the realm of the military profession, grading systems also define the hierarchy among officers and enlisted personnel. The ranking order of military graduation classes also drives the selection process to future commissions, with professional, familiar and financial implications. Traditional method of student achievement evaluation only use arithmetic mean and convert them to rankings, but this does not distinguish the effective merit of similar grades. In the present study, a fuzzy logic-based model was developed in order to classify the student grades according to a merit scale. The box plot distribution of student grades is the basis to build the merit scale fuzzy sets. Data sets were categorized into seven fuzzy sets using the box plot. This method was applied in a hypothetical database of 40 students in ten disciplines. In order to test the new method, the database of the first six students got the same score. Results of this study showed the efficiency of the proposed method when compared to traditional ones.
\end{abstract}

Keywords: $\quad$ Fuzzy grading system; student evaluation; merit; box plot. 


\section{INTRODUÇÃO}

O método recebe a denominação de "composição" pois se fundamenta na utilização combinada de três metodologias distintas como ferramentas de modelagem. Primeiro, utiliza os fundamentos de agrupamentos não hierárquicos (clusters) para classificar os atributos de desempenho das diferentes alternativas em problemas multicritério. Segundo, esses agrupamentos são particionados em torno de parâmetros estatísticos de box plot, desenhados para a base de dados formada pelos atributos. Terceiro, as distâncias Euclidianas desses atributos aos centros dos agrupamentos, definidos pelo box plot, são associadas a funções de pertinências de conjuntos fuzzy, a serem convertidas em atributos relativos por sistema de inferência fuzzy. Esse termo "relativo" se refere à criação de um novo atributo, que deixa de ser um valor absoluto da alternativa na escala de avaliação de desempenho, segundo um critério, para ser um novo valor relativo, que considera a sua posição no box plot do mesmo critério.

O caso de aplicação mais evidente da "Composição Relativa de Atributos" (CRA) ocorre em escalas de avaliação de desempenho acadêmico. Entretanto, os sistemas de apoio à decisão em gestão de recursos humanos, modelados para classificar desempenho em processos de admissão, seleção e promoção do pessoal, também se configuram com elevado potencial de aplicação. No caso específico da profissão militar, os sistemas de classificação também definem a hierarquia nas turmas de oficiais e praças e, por vezes, a prioridade de escolha de futuras comissões, com implicações profissionais, familiares e financeiras. A diversidade de processos de avaliação do desempenho do pessoal durante a carreira militar pode-se beneficiar do uso de métodos quantitativos que valorizem a meritocracia, de forma a gerar uma classificação mais justa e próxima da realidade. Também visualizam-se aplicações em avaliação de desempenho de processos e serviços.

Para ilustrar a diferença conceitual entre um atributo "absoluto" e um atributo "relativo", recorre-se a um problema hipotético de avaliação acadêmica. Supõe-se que um aluno "A" obteve a nota "10" na disciplina "X" e um aluno "B" obteve a mesma nota "10" na disciplina "Y", ambas consideradas em escala de " 0 " a "10". No entanto, a disciplina "X" apresentou significativo grau de dificuldade durante o período letivo, de forma que as notas da maioria dos alunos na disciplina " $X$ " se concentraram na faixa entre " 0 " e " 3 ". Nesse contexto, admite-se que o aluno "A" obteve um excepcional desempenho.

Por outro lado, a disciplina " $Y$ " foi a mais fácil do período letivo e suas notas se concentraram entre " 8 " e " 10 ". O aluno "B" obteve a nota máxima nessa disciplina, embora a maioria dos demais alunos também tenha obtido um ótimo desempenho. Esse caso torna evidente que o "mérito relativo" da mesma nota "10" apresenta significativa diferença para retratar o desempenho dos dois alunos, com maior mérito ao aluno "A". De fato, o único aspecto em comum entre essas disciplinas é a escala de "0" a " $10 "$ ", pois normalmente são ministradas por docentes diferentes, além de envolverem assuntos, bibliografias e níveis de dificuldade distintos. Nesse contexto, o uso de um fator de conversão desses atributos permitiria diferenciar as notas absolutamente equivalentes a "10" dos alunos "A" e "B", distinguindo assim o mérito relativo entre esses alunos. As notas "10" são atributos "absolutos", enquanto os novos atributos obtidos a partir da aplicação do método proposto são "relativos".

A CRA foi aplicada em uma base de dados hipotética, composta por notas de 40 alunos em dez disciplinas, totalizando 400 diferentes atributos para a análise. Com a finalidade de testar a eficácia do método, aos seis alunos com melhores médias finais no período letivo foram atribuídas as mesmas médias e, para os três primeiros alunos, as mesmas notas absolutas, apenas distribuídas por disciplinas diferentes. Dessa forma, o método mostrou-se eficaz ao distinguir o mérito dos seis primeiros classificados, com o desafio adicional de diferenciar as notas iguais aos três primeiros alunos. Ao final, cada um desses seis alunos obteve médias finais diferentes, por conversão de suas notas absolutas em fatores 
de correção que permitiram distinguir o de melhor desempenho relativo dentre seus pares.

A finalidade desse artigo é apresentar a CRA como um fator de conversão em escalas de avaliação de desempenho, capaz de valorizar o mérito de uma determinada nota ao considerar sua posição relativa à distribuição das demais na mesma disciplina. $\mathrm{O}$ artigo foi delineado nos itens a seguir: (2) revisão da literatura, com os principais aspectos teóricos da estatística por box plot e da lógica fuzzy aplicada a sistemas de classificação; (3) a metodologia utilizada para o cálculo da CRA, incluindo a aplicação em um estudo de caso hipotético em turma de 40 alunos; (4) a análise dos resultados e, por fím, no item (5) a conclusão do artigo.

\section{REVISÃO DA LITERATURA}

Desde a proposta de Zadeh (1965) sobre a teoria dos conjuntos fuzzy em 1965, diversas novas teorias e aplicações surgiram, nos mais variados campos do conhecimento humano. O portal da Universidade de Berkeley sobre as iniciativas em computação soft, que enquadra a lógica fuzzy, traz números relevantes que evidenciam esse aspecto. Dentre outros, cabem destacar o registro de mais de 130 mil patentes de aplicação da lógica fuzzy, em sua maioria na China, EUA e Japão, a criação de 28 periódicos internacionais especialmente dedicados à lógica fuzzy e mais de 50 mil citações ao artigo original de Zadeh (1965) no Google Scholar (BISC, 2014).

Nos últimos anos, alguns pesquisadores se dedicaram à aplicação da lógica fuzzy em sistemas de avaliação de desempenho acadêmico. Chang \& Sun (1993) desenvolveram um método para avaliação de aprendizagem para estudantes do primeiro ano do ensino médio. Chiang \& Lin (1994) elaboraram um método estatístico para apoio à qualidade do ensino dos docentes. Echauz \& Vachtsevanos (1995) apresentaram um sistema de classificação com base na performance de discentes e docentes. Biswas (1995) publicou dois métodos de avaliação acadêmica. Law (1996) incluiu em seu método a avaliação individual e de grupos de estudantes. Cheng \& Yang (1998) pesquisaram sobre o grau de subjetividade de docentes em seus processos de avaliação. Chen \& Lee (1999) aprofundaram a pesquisa de Biswas (1995).

Ma \& Zhou (2000) apresentaram um método que explora a percepção de docentes e discentes sobre o próprio processo de avaliação da aprendizagem. Wu (2003) propõe um método com menor quantidade de avaliações. Wang \& Chen (2006a) publicaram dois métodos de avaliação de questões de exames de discentes. Wang \& Chen (2006b) trouxeram novos métodos com a inclusão de graus de confiança aos números fuzzy. Bai \& Chen (2008a) apresentaram um método para avaliação de aprendizagem de discentes, com base no grau de dificuldade, relevância e complexidade de questões de exames aplicados aos estudantes. Posteriormente, Bai \& Chen (2008b) publicaram novo método para conversão automática de funções de pertinência dos graus obtidos pelos estudantes.

Cabe observar que a maioria das pesquisas publicadas sobre aplicações da lógica fuzzy em sistemas de classificação acadêmica é de autores chineses, indicando uma tendência daquela sociedade no desenvolvimento de novos métodos de distinção do mérito. Identificar os melhores valores acadêmicos contribui para alavancar a educação e, consequentemente, o desenvolvimento científico e tecnológico.

\section{MÉTODO APLICADO}

A proposta desse artigo compõe a base da tese dos autores, sendo original no campo das aplicações fuzzy em sistemas de classificação aplicadas a escalas de desempenho. A principal peculiaridade do método consiste em associar o gráfico box plot, uma forma de análise exploratória de dados originalmente proposta por Tukey (1970), a clusters e funções de pertinência fuzzy. Uma característica peculiar do box plot é distinguir pontos fora da curva, também denominados de "outliers", que podem estar associados a desempenhos excepcionais 
em escalas de avaliação de desempenho. No caso de processos de classificação acadêmica, esse aspecto é de especial significância, pois permitirá selecionar os candidatos ou discentes com performance única dentre os demais.

O método utiliza o box plot por assumir a premissa de que as notas das diversas disciplinas de uma turma de estudantes raramente configuram uma distribuição normal. Segundo Campos (2014), curvas assimétricas não admitem desvio-padrão, pois, por definição, "desvio-padrão é o ponto de inflexão da curva normal, em número de dois e simétricos em relação à média da distribuição". Este autor também destaca que "curvas assimétricas, mesmo que tenham pontos de inflexão, como possuem muitas outras curvas matemáticas, eles dificilmente seriam simétricos em relação à média". Nesse contexto, não faria sentido apoiar o método sobre os parâmetros estatísticos típicos de uma distribuição normal.

Em relação às distribuições assimétricas, o método faz uso de medianas e quartis para distribuir as notas finais da base de dados, ao invés de médias e desvios-padrão. A metodologia para o cálculo da CRA seguiu o esquema da Figura 1, com as seis etapas apresentadas nos próximos tópicos.

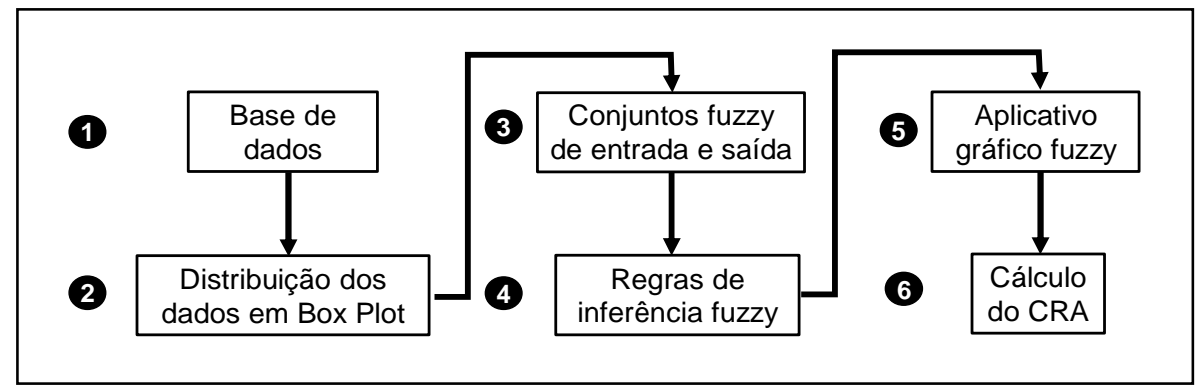

FIGURA 1: Metodologia de cálculo da CRA. Fonte: Próprios autores.

\subsection{BASE DE DADOS}

Uma base de dados contendo atributos quantitativos, referentes à avaliação de desempenho de alternativas segundo critérios, nos termos de problemas multicritério, constitui o ponto inicial para a aplicação da CRA. Dessa forma, para o método não há o interesse nos processos ou métodos que antecedem à produção da base de dados.

O processo pode receber dois tipos de tratamento preliminar na base de dados: normalizar as escalas em cada critério e/ou atribuir pesos aos diferentes critérios. Esses passos não foram aplicados no estudo de caso hipotético, pois as escalas de notas entre zero e dez já estão normalizadas e por não é comum a atribuição de pesos às diferentes disciplinas acadêmicas em períodos letivos. Entretanto, esses procedimentos poderiam compor etapas preliminares à aplicação da CRA.

Uma base de dados hipotética foi utilizada para ilustrar a aplicação do método. Em uma escola, ao término do período letivo, uma turma de 40 alunos obteve os seguintes graus de uma escala de avaliação de aprendizagem, distribuída entre os valores zero e dez, em dez disciplinas, denominadas "A", "B", "C", "D", "E", "F", "G", "H", "I" e "J". Essa base de dados inicial encontra-se ordenada por classificação das médias finais, calculados por média aritmética das notas obtidas pelos alunos nas dez disciplinas, conforme a Tabela 1. 
Tabela 1 - Base de dados

\begin{tabular}{|c|c|c|c|c|c|c|c|c|c|c|c|}
\hline & $\mathbf{A}$ & B & $\mathbf{C}$ & D & $\mathbf{E}$ & $\mathbf{F}$ & $\mathbf{G}$ & $\mathbf{H}$ & I & $\mathbf{J}$ & Médias finais \\
\hline 1 & 10 & 9.6 & 8.8 & 8.5 & 9.5 & 9.1 & 8.9 & 9.3 & 8.6 & 10 & 9.23 \\
\hline 2 & 8.9 & 8.6 & 9.1 & 9.3 & 10 & 10 & 8.8 & 8.5 & 9.5 & 9.6 & 9.23 \\
\hline 3 & 9.5 & 8.8 & 9.3 & 10 & 9.1 & 8.6 & 8.5 & 9.6 & 10 & 8.9 & 9.23 \\
\hline 4 & 10 & 9.8 & 7.8 & 8.2 & 10 & 10 & 8.4 & 8.1 & 10 & 10 & 9.23 \\
\hline 5 & 9.9 & 9.4 & 9.8 & 9.6 & 9.1 & 8.8 & 9.3 & 8.2 & 9.2 & 9 & 9.23 \\
\hline 6 & 10 & 10 & 9.2 & 9.3 & 9.7 & 10 & 10 & 9.3 & 8 & 6.8 & 9.23 \\
\hline 7 & 9.3 & 8.4 & 8.2 & 8.6 & 9.2 & 9.5 & 9.1 & 9.3 & 8.8 & 9.4 & 8.98 \\
\hline 8 & 3.2 & 10 & 7.1 & 8.2 & 10 & 9.1 & 10 & 9 & 9 & 10 & 8.56 \\
\hline 9 & 8.2 & 9 & 8 & 7.7 & 6.8 & 7.1 & 8 & 8.1 & 10 & 10 & 8.29 \\
\hline 10 & 8 & 7.3 & 8.3 & 6.7 & 7.3 & 8 & 8.3 & 9 & 10 & 9.3 & 8.22 \\
\hline 11 & 6.5 & 8 & 9 & 9.6 & 7 & 6.2 & 7.1 & 8.1 & 10 & 10 & 8.15 \\
\hline 12 & 9.2 & 8 & 8 & 6 & 6.9 & 5.5 & 6.8 & 9.3 & 10 & 10 & 7.97 \\
\hline 13 & 6.2 & 8 & 7.4 & 6.2 & 7.3 & 7 & 7 & 9.9 & 10 & 10 & 7.9 \\
\hline 14 & 3.7 & 8.2 & 9.2 & 6.8 & 7.3 & 9.1 & 6.9 & 8.2 & 9.1 & 10 & 7.85 \\
\hline 15 & 9.8 & 7 & 7.9 & 9.5 & 4.8 & 6.9 & 7 & 10 & 7 & 8 & 7.79 \\
\hline 16 & 6.9 & 7.2 & 7.1 & 5.8 & 7 & 8 & 7.9 & 8 & 10 & 9.7 & 7.76 \\
\hline 17 & 8 & 6.4 & 9.3 & 7.5 & 3 & 6 & 7 & 9.5 & 10 & 10 & 7.67 \\
\hline 18 & 6.3 & 7.3 & 7.4 & 9 & 8.2 & 6.7 & 6.4 & 10 & 7.6 & 7.5 & 7.64 \\
\hline 19 & 7.3 & 8.3 & 9.3 & 6.3 & 5.7 & 4 & 6.4 & 8 & 10 & 10 & 7.53 \\
\hline 20 & 1.2 & 8.9 & 7.8 & 7 & 5.5 & 9 & 7.5 & 9.2 & 9 & 10 & 7.51 \\
\hline 21 & 7.1 & 7.6 & 9 & 7.8 & 6 & 5.2 & 7.5 & 8 & 10 & 5.9 & 7.41 \\
\hline 22 & 5.1 & 8 & 8.4 & 5.4 & 5.6 & 7.7 & 8.2 & 10 & 8 & 7 & 7.34 \\
\hline 23 & 7.4 & 8 & 9.1 & 5.5 & 8.1 & 3.4 & 7.8 & 10 & 7 & 7 & 7.33 \\
\hline 24 & 6.2 & 7.1 & 9.6 & 6 & 5.5 & 7 & 6 & 8.2 & 7.6 & 10 & 7.32 \\
\hline 25 & 6.8 & 7.6 & 9.3 & 7.3 & 6 & 3.5 & 7.5 & 8 & 10 & 6.8 & 7.28 \\
\hline 26 & 2.5 & 8 & 7.9 & 5 & 7 & 7.8 & 8 & 8.5 & 10 & 8 & 7.27 \\
\hline 27 & 4.2 & 6.9 & 9.5 & 6 & 6.5 & 2 & 8.5 & 9 & 10 & 10 & 7.26 \\
\hline 28 & 9 & 8.4 & 7.9 & 6.3 & 3.2 & 5.8 & 7 & 10 & 10 & 5 & 7.26 \\
\hline 29 & 2.8 & 7.5 & 8.3 & 7 & 7.4 & 7 & 6.8 & 10 & 7 & 8.3 & 7.21 \\
\hline 30 & 5.5 & 6.9 & 8 & 7 & 5.9 & 5.5 & 6.9 & 8.9 & 7.3 & 10 & 7.19 \\
\hline 31 & 6 & 7.7 & 9 & 5 & 5.2 & 6.5 & 7.2 & 8.3 & 10 & 6.4 & 7.13 \\
\hline 32 & 4.2 & 6.1 & 7.4 & 5.2 & 6.2 & 6.5 & 8 & 8.4 & 10 & 9 & 7.1 \\
\hline 33 & 3 & 8.2 & 8.8 & 6.8 & 8 & 5.4 & 6 & 10 & 7 & 7 & 7.02 \\
\hline 34 & 4.3 & 8.1 & 9.2 & 7.8 & 7 & 1.8 & 6.8 & 10 & 9 & 5 & 6.9 \\
\hline 35 & 1.4 & 7.5 & 8.3 & 8 & 6.4 & 4.5 & 6.6 & 10 & 10 & 5.6 & 6.83 \\
\hline 36 & 3.8 & 8 & 6.8 & 6.4 & 3 & 7.8 & 7.2 & 9.5 & 10 & 5 & 6.75 \\
\hline 37 & 1.3 & 8.2 & 9 & 5.3 & 4.3 & 5.3 & 7.2 & 9.4 & 10 & 7.5 & 6.75 \\
\hline 38 & 2.8 & 6.8 & 10 & 5 & 4 & 3.4 & 6.8 & 10 & 9.5 & 9 & 6.73 \\
\hline 39 & 7.6 & 6 & 9 & 6 & 4.9 & 3.2 & 7.2 & 9 & 7 & 6 & 6.59 \\
\hline 40 & 3.3 & 7.4 & 10 & 5.5 & 4.9 & 2.2 & 7 & 8 & 10 & 6 & 6.43 \\
\hline
\end{tabular}

Fonte: Próprios autores.

Nesta base de dados, verifica-se que os seis primeiros alunos obtiveram a mesma média final (i.e. 9,23), sendo que os três primeiros lograram as mesmas notas, apenas distribuídas por disciplinas diferentes. Esse cenário desfavorável para a distinção do mérito 
entre os alunos foi explorado com a finalidade de apresentar a utilidade do método proposto.

\subsection{DISTRIBUIÇÃO DOS DADOS EM BOX PLOT}

O box plot foi a ferramenta utilizada para identificar e diferenciar o valor de cada nota em cada disciplina. De certa forma, é possível associar as diferentes notas ao problema de paridade do poder de compra (PPC) entre unidades monetárias. Em escala de zero a dez unidades monetárias, um determinado produto pode custar dez reais na fábrica "A", dez euros na fábrica "B" e dez dólares na fábrica "C". Embora representem valores numéricos iguais, os valores monetários diferentes impedem o uso de uma média aritmética para agregar esses preços, que teria como resultado dez unidades de uma moeda híbrida e inexistente. No caso das moedas, o mercado precifica essas cotações e atribui as fórmulas de conversão ou taxas de câmbio utilizadas nos diversos países. No caso das notas das diferentes disciplinas dessa pesquisa, o box plot foi a ferramenta utilizada para diferenciar o "valor" das notas, que distribui os atributos por quartis, a partir de partições em torno da mediana.

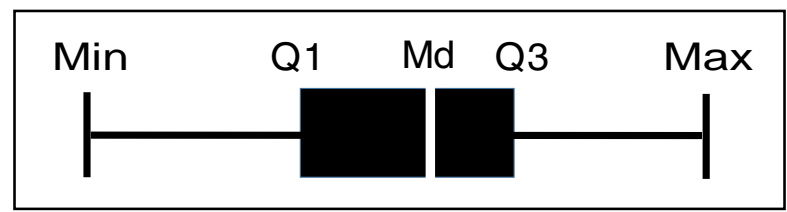

FIGURA 2: Gráfico box plot. Fonte: Adaptado de Freund \& Simon (2000).

Os gráficos box plot são referenciados pela mediana da distribuição, o que permite a sua aplicação nos mais variados tipos de assimetrias de dados. A Figura 2 apresenta um gráfico box plot genérico: valor mínimo dos dados (Min), valor do $1^{\circ}$ quartil (Q1), que reúne $25 \%$ dos dados, valor da mediana (Md), que distribui a quantidade de dados em dois grupos, valor do $3^{\circ}$ quartil (Q3), que reúne $75 \%$ dos dados e o valor máximo dos dados (Max).

$\mathrm{O}$ valor das notas em cada disciplina, tal qual o exemplo do PPC das moedas, pode ser verificado graficamente por meio das distribuições box plot, conforme a Figura 3. É interessante verificar que as diferentes formas dos box plot caracterizam "famílias" de distribuição distintas, com graus variados de assimetria. Esse aspecto fundamenta a proposta do método CRA em considerar cada disciplina com um valor diferente, em decorrência de sua distribuição.

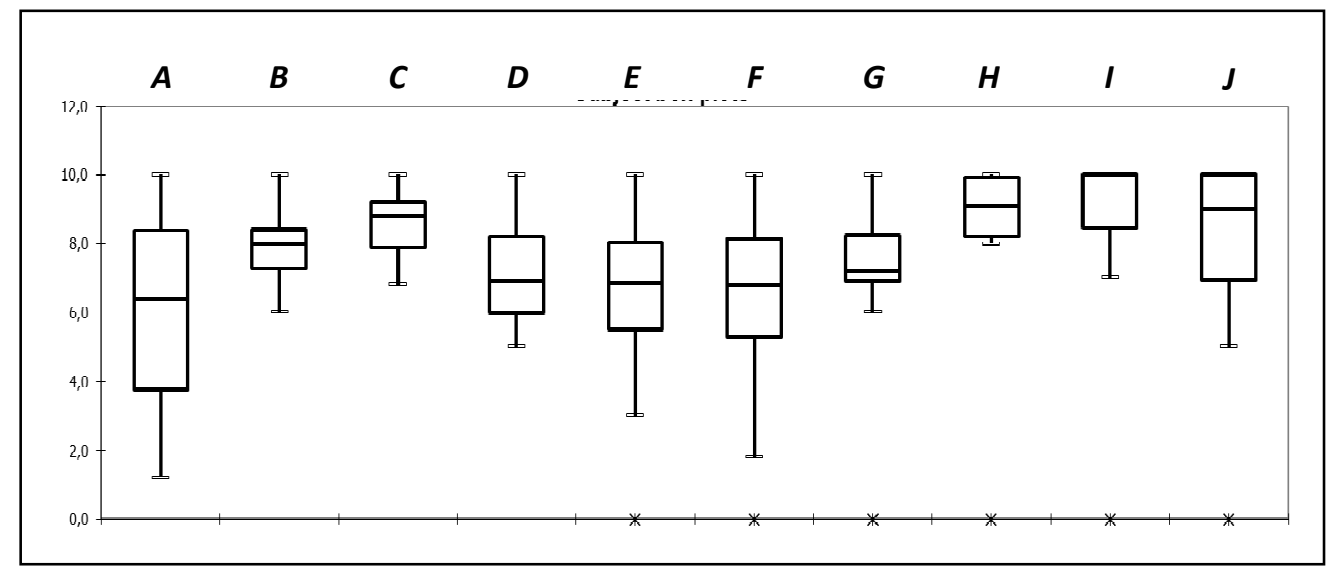

FIGURA 3: Distribuição das notas das disciplinas em box plot. Fonte: Próprios autores.

Na Figura 3, uma nota "6,0" na disciplina "A" encontra-se próxima da mediana, enquanto outra nota idêntica na disciplina "B" encontra-se entre as menores. O posicionamento destas notas em cada distribuição possui méritos diferentes, pois representam desempenhos diferentes. Assim, não parece meritório que a nota da disciplina "A" seja 
diretamente agregada com a da disciplina "B". Esse é o argumento que fundamenta a proposta do método CRA.

A Figura 4 apresenta, na porção inferior, uma escala com seis intervalos e sete parâmetros, associada ao box plot em posição horizontal, que inclui os limites da escala de avaliação, "0" e "10", do limite inferior (LI) e do limite superior (LS). O LI e o LS são obtidos com base na amplitude interquartílica (DQ). A DQ equivale ao Q3 menos Q1. O LI equivale ao Q1 menos 1,5 vezes DQ. O LS equivale ao Q3 mais 1,5 vezes DQ, conforme o modelo proposto por Tukey (1970). Esses limites LI e LS demarcam a faixa de "pontos fora da curva", tanto negativos quanto positivos. Os parâmetros "0", LI, Q1, Md, Q3, LS e "10" representam os centros de seus respectivos clusters, formados pelos intervalos das escalas ao redor de seus valores. Dessa forma, esses parâmetros configuram os valores de máxima pertinência dos conjuntos fuzzy de entrada do sistema.

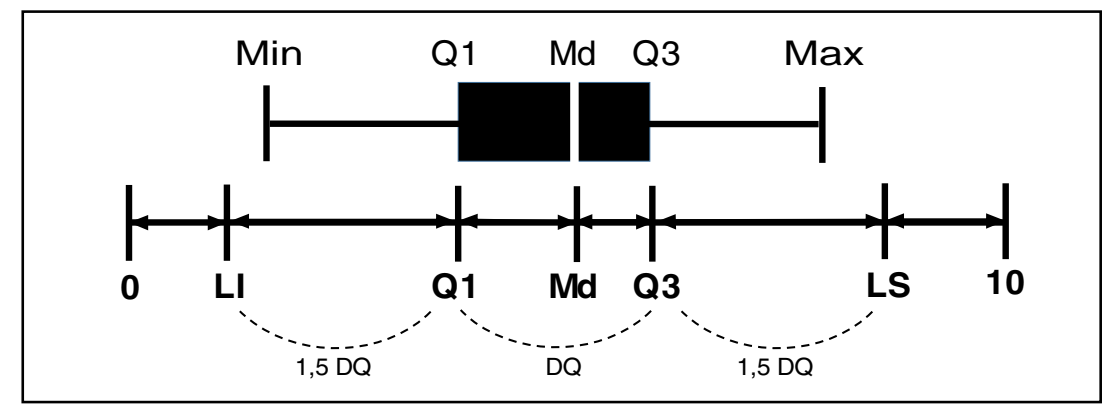

FIGURA 4: Outros parâmetros em gráficos box plot. Fonte: Adaptado de Freund \& Simon (2000).

Os parâmetros são calculados a partir das distribuições das notas da base de dados, com auxílio do software MS Excel, conforme a Tabela 2. Este aplicativo traz as fórmulas de cálculo da mediana e demais quartis, simplificando a produção dos parâmetros. Os valores inferiores e superiores aos limites da escala serão respectivamente aproximados a " 0 " e " 10 ", por ocasião da criação dos conjuntos fuzzy, em processo a ser descrito na próxima etapa.

Tabela 2 - Parâmetros box plot

\begin{tabular}{|l|c|c|c|c|c|c|c|c|c|c|}
\hline & A & B & C & D & E & F & G & H & I & J \\
\hline LI (Q1-1,5 DQ) & $-3,775$ & 5,650 & 5,950 & 2,700 & 1,638 & 0,513 & 4,838 & 5,538 & 6,125 & 2,375 \\
\hline Q1 & 3,725 & 7,300 & 7,900 & 6,000 & 5,500 & 5,275 & 6,900 & 8,200 & 8,450 & 6,950 \\
\hline Q2 (mediana) & 6,400 & 8,000 & 8,800 & 6,900 & 6,850 & 6,800 & 7,200 & 9,100 & 10,000 & 9,000 \\
\hline Q3 & 8,725 & 8,400 & 9,200 & 8,200 & 8,075 & 8,450 & 8,275 & 9,975 & 10,000 & 10,000 \\
\hline DQ & 5,000 & 1,100 & 1,300 & 2,200 & 2,575 & 3,175 & 1,375 & 1,775 & 1,550 & 3,050 \\
\hline LS (Q3+1,5DQ) & 16,225 & 10,050 & 11,150 & 11,500 & 11,938 & 13,213 & 10,338 & 12,638 & 12,325 & 14,575 \\
\hline
\end{tabular}

Fonte: Próprios autores.

\subsection{CONJUNTOS FUZZY DE ENTRADA E SAÍDA}

A partir dos parâmetros box plot referentes a cada disciplina, é possível elaborar os conjuntos fuzzy de entrada, sob a forma de funções triangulares, conforme a Figura 5. O uso da função de pertinência triangular para cada conjunto fuzzy atende à premissa do modelo proposto, qual seja considerar as máximas pertinências às notas iguais aos parâmetros " 0 ”, LI, Q1, Md, Q3, LS e "10". Isso não significa dizer que as notas, por exemplo, entre Q1 e Q3 apresentem uma distribuição triangular, mas a compreensão de que uma nota igual a Q1 ou Q3 apresenta pertinência nula ao conjunto fuzzy "Mediano", enquanto uma nota igual à mediana apresenta pertinência máxima a esse conjunto. Outras formas de representar as funções de pertinência (i.e. trapezoidal, senoidal, gaussiana, dentre outras) podem ser utilizadas, entretanto a opção por funções triangulares privilegia a simplicidade de 
interpretação das variáveis linguísticas dos conjuntos fuzzy, simplifica a construção gráfica dos conjuntos fuzzy, produz resultados satisfatórios e atende eficazmente ao propósito do método.

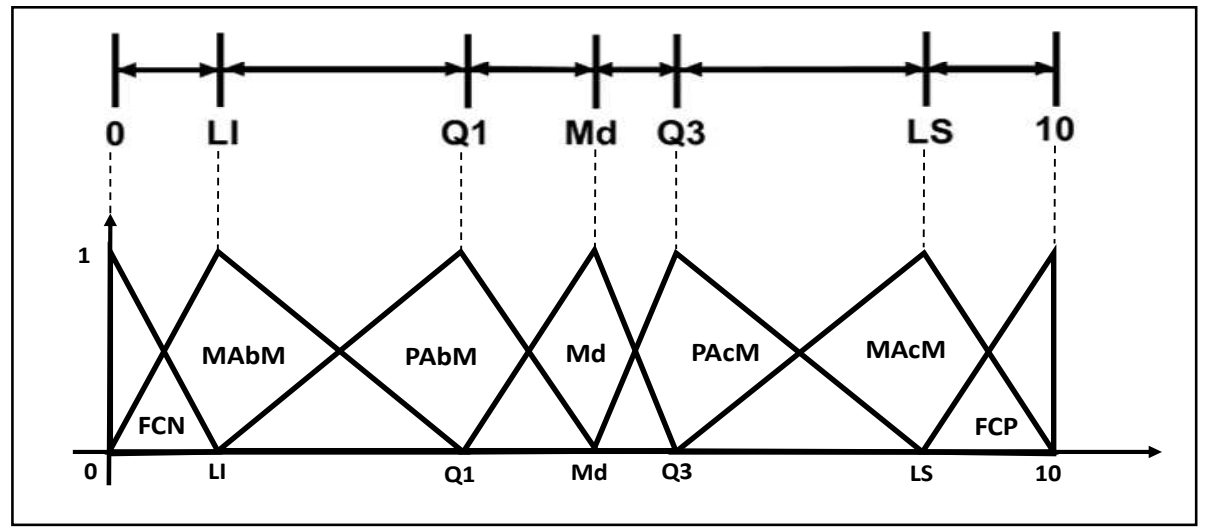

FIGURA 5: Conjuntos fuzzy de entrada. Fonte: Próprios autores.

Os conjuntos fuzzy com máxima pertinência nos extremos da escala são, respectivamente, "Fora da Curva Negativo" (FCN) e "Fora da Curva Positivo" (FCP). O conjunto com máxima pertinência sobre a mediana se denomina "Mediano" (Md). A seguir, são traçados os conjuntos "Muito Acima da Mediana" (MAcM) e "Pouco Acima da Mediana" (PAcM), centrados sobre o LS e o Q3, respectivamente. Finalmente, para o setor inverso, são elaborados os conjuntos "Muito Abaixo da Mediana" (MAbM) e "Pouco Abaixo da Mediana" (PAbM), centrados sobre o LI e o Q1, respectivamente.

Dependendo do grau de assimetria da distribuição dos dados, é possível que alguns desses conjuntos sejam eliminados durante o processo de criação dos conjuntos fuzzy. Por exemplo, as distribuições com forte assimetria à esquerda, onde os dados estão concentrados no topo da escala, provavelmente resultarão em valores do LS e talvez do Q3 acima da nota "10". Nesse caso, o LS e o Q3 seriam eliminados, pois somente devem existir conjuntos fuzzy para os parâmetros com valores entre os limites da escala (i.e. "0" e "10").

Esses casos especiais de eliminação de conjuntos apresentam coerência racional, pois uma forte assimetria à esquerda não induz à existência de notas no conjunto "Fora da Curva Positivo". Se a maioria dos alunos obteve notas elevadas, existe menor mérito relativo aos que obtiveram nota " 10 " do que sob assimetria oposta. O mesmo ocorreria nos casos de assimetria à direita, onde as notas se concentram próximo do "0", para aqueles que obtiveram notas mais baixas. Esses casos especiais serão explorados no caso hipotético apresentado neste artigo.

A Tabela 3 resume os valores de máxima pertinência das funções triangulares do estudo de caso. A regra prática para obter esses valores é substituir os valores negativos dos parâmetros apresentados na Tabela 2 pelo mínimo da escala de desempenho, no caso " 0 " e os valores superiores ao máximo da escala de desempenho, no caso " 10 ”, por " 10 ":

Tabela 3 - Máxima pertinência dos conjuntos fuzzy de entrada

\begin{tabular}{|l|c|c|c|c|c|c|c|c|c|c|}
\hline Conj Fuzzy & A & B & C & D & E & F & G & H & I & J \\
\hline Fora da curva Neg & 0,00 & 0,00 & 0,00 & 0,00 & 0,00 & 0,00 & 0,00 & 0,00 & 0,00 & 0,00 \\
\hline Muito abaixo Md & 0,00 & 5,65 & 5,95 & 2,70 & 1,64 & 0,51 & 4,84 & 5,54 & 6,13 & 2,38 \\
\hline Pouco abaixo Md & 3,73 & 7,30 & 7,90 & 6,00 & 5,50 & 5,28 & 6,90 & 8,20 & 8,45 & 6,95 \\
\hline Mediano & 6,40 & 8,00 & 8,80 & 6,90 & 6,85 & 6,80 & 7,20 & 9,10 & 10,00 & 9,00 \\
\hline Pouco acima Md & 8,73 & 8,40 & 9,20 & 8,20 & 8,08 & 8,45 & 8,28 & 9,98 & 10,00 & 10,00 \\
\hline Muito acima Md & 10,00 & 10,00 & 10,00 & 10,00 & 10,00 & 10,00 & 10,00 & 10,00 & 10,00 & 10,00 \\
\hline Fora da curva Pos & 10,00 & 10,00 & 10,00 & 10,00 & 10,00 & 10,00 & 10,00 & 10,00 & 10,00 & 10,00 \\
\hline
\end{tabular}

Fonte: Próprios autores. 
Esses valores dão origem aos conjuntos fuzzy de entrada, que correspondem a funções de pertinência triangulares do tipo $[a, b, c]$, onde " $a$ " e "c" são os vértices da base do triângulo, representando a pertinência nula, enquanto "b" é o vértice, com pertinência máxima, nesse caso "1". A Tabela 4 delineia os parâmetros dos conjuntos fuzzy de entrada, onde as marcações " $\mathrm{x}$ " indicam os conjuntos eliminados:

Tabela 4 - Conjuntos fuzzy de entrada

\begin{tabular}{|c|c|c|c|c|c|c|c|c|c|c|c|c|c|c|c|c|c|c|c|c|c|}
\hline \multirow[t]{2}{*}{ Disc. } & \multicolumn{3}{|c|}{ FCN } & \multicolumn{3}{|c|}{ MAbM } & \multicolumn{3}{|c|}{ PAbM } & \multicolumn{3}{|c|}{ Mediano } & \multicolumn{3}{|c|}{ PAcM } & \multicolumn{3}{|c|}{ MAcM } & \multicolumn{3}{|c|}{ FCP } \\
\hline & a & b & c & a & b & c & a & b & c & $a$ & b & c & a & b & c & a & b & c & $a$ & b & c \\
\hline A & $x$ & $x$ & $x$ & 0,00 & 0,00 & 3,73 & 0,00 & 3,73 & 6,40 & 3,73 & 6,4 & 8,73 & 6,4 & 8,73 & 10 & 8,73 & 10 & 10 & $x$ & $x$ & $x$ \\
\hline$B$ & 0,00 & 0,00 & 5,65 & 0,00 & 5,65 & 7,30 & 5,65 & 7,30 & 8,00 & 7,30 & 8,00 & 8,4 & 8,00 & 8,4 & 10 & 8,4 & 10 & 10 & $x$ & $x$ & $x$ \\
\hline D & 0,00 & 0,00 & 2,70 & 0,00 & 2,70 & 6,00 & 2,70 & 6,00 & 6,90 & 6,00 & 6,9 & 8,2 & 6,9 & 8,2 & 10 & 8,2 & 10 & 10 & $\mathrm{x}$ & $\mathrm{x}$ & $\mathrm{x}$ \\
\hline $\mathrm{E}$ & 0,00 & 0,00 & 1,64 & 0,00 & 1,64 & 5,50 & 1,64 & 5,50 & 6,85 & 5,50 & 6,85 & 8,08 & 6,85 & 8,08 & 10 & 8,08 & 10 & 10 & $x$ & $x$ & $x$ \\
\hline $\mathrm{F}$ & 0,00 & 0,00 & 0,51 & 0,00 & 0,51 & 5,28 & 0,51 & 5,28 & 6,80 & 5,28 & 6,8 & 8,45 & 6,8 & 8,45 & 10 & 8,45 & 10 & 10 & $\mathrm{x}$ & $x$ & $\mathrm{x}$ \\
\hline 1 & 0,00 & 0,00 & 6,13 & 0,00 & 6,13 & 8,45 & 6,13 & 8,45 & 10,00 & 8,45 & 10 & 10 & $x$ & $x$ & $\mathrm{x}$ & $\mathrm{x}$ & $x$ & $x$ & $x$ & $x$ & $x$ \\
\hline $\mathrm{J}$ & 0,00 & 0,00 & 2,38 & 0,00 & 2,38 & 6,95 & 2,38 & 6,95 & 9,00 & 6,95 & 9 & 10 & 9 & 10 & 10 & $x$ & $x$ & $x$ & $x$ & $x$ & $\mathrm{x}$ \\
\hline
\end{tabular}

Fonte: Próprios autores.

Os conjuntos fuzzy de saída do sistema são distribuídos por sete faixas, divididos igualmente sobre a escala de " 0 " a "10", conforme a Figura 6. Os conjuntos fuzzy foram denominados Insuficiente (I), Ruim (Ru), Regular (Rg), Bom (B), Muito Bom (MB), Ótimo (O) e Excepcional (E).

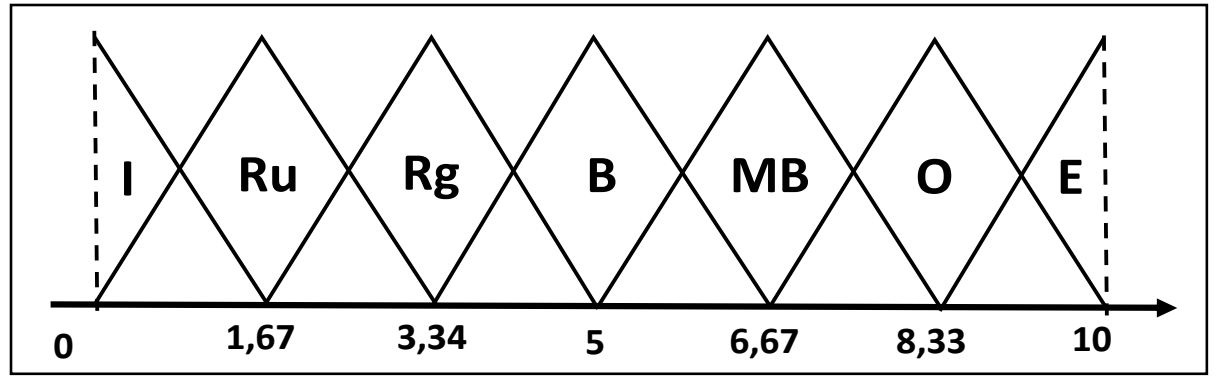

FIGURA 6: Conjuntos fuzzy de saída. Fonte: Próprios autores.

\subsection{REGRAS DE INFERÊNCIA FUZZY}

A correlação entre a mesma quantidade de conjuntos fuzzy de entrada e saída, sendo sete conjuntos sob condições normais e inferior a sete sob condições especiais, conforme descritos na etapa anterior, indica o emprego do método de rápida inferência, denominado "Union Rules ConFiguration" (URC), desenvolvido por Combs (1997).

Nesse contexto, as seguintes regras de inferência do tipo "se-então" encontram-se previstas para o caso máximo de sete conjuntos fuzzy de entrada e os correspondentes sete conjuntos fuzzy de saída. As regras de inferência refletem a simplicidade do modelo e a racionalidade da associação entre as notas com distribuição FCP ao conjunto "excepcional", seguindo o mesmo procedimento aos demais conjuntos.

(1) Se a nota é "Fora da curva negativo" então a CRA é "Insuficiente"; ou

(2) Se a nota é "Muito abaixo da mediana" então a CRA é "Ruim"; ou

(3) Se a nota é "Pouco abaixo da mediana" então a CRA é "Regular"; ou

(4) Se a nota é "Mediana" então a CRA é "Bom"; ou

(5) Se a nota é "Pouco acima da mediana" então a CRA é "Muito Bom"; ou

(6) Se a nota é "Muito acima da mediana" então a CRA é "Ótimo"; ou

(7) Se a nota é "Fora curva positivo" então a CRA é "Excepcional". 


\subsection{APLICATIVO GRÁFICO FUZZY}

O sistema de inferência fuzzy torna-se operacionalmente mais simples para modelar e aplicar quando programado em softwares de apoio, preferencialmente dotados de interface gráfica para a elaboração de sistemas fuzzy. No caso dessa pesquisa, foi utilizado o MATLAB R2013a, com suporte do aplicativo Fuzzy Toolbox.

\subsection{CÁlCULO DA CRA}

A aplicação do CRA sobre as notas dos seis alunos com maiores médias gerou os resultados da Tabela 5 .

Tabela 5 - Resultado do cálculo da CRA

\begin{tabular}{|c|c|c|c|c|c|c|c|c|c|c|c|c|}
\hline Al./Disc. & A & B & C & D & E & F & G & H & I & J & Médias CRA & Classif \\
\hline $\mathbf{1}$ & 8,33 & 7,85 & 5 & 7,01 & 7,83 & 7,39 & 7,31 & 5,45 & 3,55 & 6,66 & $\mathbf{6 , 6 4}$ & 3 \\
\hline $\mathbf{2}$ & 6,95 & 6,93 & 6,18 & 7,65 & 8,33 & 8,33 & 7,23 & 3,94 & 4,41 & 5,97 & $\mathbf{6 , 5 9}$ & 5 \\
\hline $\mathbf{3}$ & 7,64 & 7,15 & 6,93 & 8,33 & 7,54 & 6,88 & 6,94 & 5,92 & 5 & 4,88 & $\mathbf{6 , 7 2}$ & 2 \\
\hline $\mathbf{4}$ & 8,33 & 8,06 & 3,22 & 6,66 & 8,33 & 8,33 & 6,82 & 3,25 & 5 & 6,66 & $\mathbf{6 , 4 7}$ & 6 \\
\hline $\mathbf{5}$ & 8,15 & 7,67 & 7,85 & 7,89 & 7,54 & 7,11 & 7,62 & 3,34 & 4,14 & 5 & $\mathbf{6 , 6 3}$ & 4 \\
\hline $\mathbf{6}$ & 8,33 & 8,33 & 6,66 & 7,65 & 8,01 & 8,33 & 8,33 & 5,45 & 2,94 & 3,26 & $\mathbf{6 , 7 3}$ & 1 \\
\hline
\end{tabular}

Fonte: Próprios autores.

Embora os seis melhores classificados apresentassem médias aritméticas iguais, a CRA permitiu distinguir os seis resultados, onde se verifica que a melhor média foi " 6,73 " e a sexta "6,47". De fato, as notas foram redistribuídas em suas respectivas faixas, elaboradas a partir dos box plot de cada disciplina e do sistema de inferência fuzzy.

\section{ANÁLISE DOS RESULTADOS}

Os resultados finais das médias aritméticas das CRA permitiram distinguir o mérito entre os seis alunos, apresentando o aluno " 6 " com o melhor desempenho dentre os demais alunos. O método tradicional de agregação por notas absolutas não permite quantificar o mérito de uma mesma nota sobre as demais. A CRA distinguiu os seis alunos, anteriormente equiparados em suas médias aritméticas. Os resultados obtidos com a aplicação da CRA indicam que o aluno " 6 " obteve suas maiores notas onde a distribuição dos demais alunos se concentrava em direção à base da escala de avaliação de desempenho, valorizando as suas notas.

Por necessidade de concisão do artigo, não foram incluídos os cálculos dos fatores referentes aos demais alunos. O diferencial do método proposto justamente reside na capacidade de distinguir o mérito relativo dos melhores candidatos ou alunos, quando suas médias aritméticas são iguais ou próximas. Dependendo das distribuições das notas em cada disciplina, é provável que haja uma inversão da classificação de candidatos ou alunos com médias aritméticas próximas. Entretanto, o "ótimo de Pareto", que indica aquele que obteve as melhores notas em todas as disciplinas será sempre a maior média aritmética e a maior CRA, assim como aquele que tenha obtido as piores notas em todas as disciplinas terá sempre a pior média e a pior CRA.

Por utilizar gráficos box plot, que refletem uma distribuição de dados, cabe ressaltar que a inclusão ou exclusão de qualquer dado, assim como uma eventual correção/revisão de uma ou mais notas, requer novo procedimento de cálculo, pois isso afeta a distribuição dos dados da disciplina. Esse aspecto confere um esforço adicional para aplicação do método. 


\section{CONCLUSÃO}

A CRA demonstrou sua eficiência em relação ao tradicional método de cálculo por média aritmética, ao ser aplicado sobre um estudo de caso com 40 alunos e suas notas em dez disciplinas, em que as médias finais dos seis primeiros alunos era igual. O método proposto assegura a distinção do discente com maior mérito relativo dentre seus concorrentes.

A existência de sistemas de classificação, para auxiliar organizações e instituições nos diversos processos de admissão, seleção e promoção do pessoal, evidencia a intenção de distinguir o mérito. Entretanto os métodos usuais de agregação das notas não refletem essa finalidade intrínseca e indissociável de um processo de classificação. Se a distinção do mérito não fosse necessária, bastava substituir o processo de classificação por um sistema eliminatório do tipo "aprovado / reprovado".

A finalidade desse artigo foi apresentar um método de classificação em escalas de avaliação de desempenho que permitisse valorizar o mérito de uma determinada nota, ao considerar sua posição relativa à distribuição das demais notas da mesma disciplina. $\mathrm{O}$ método proposto distribui as notas por gráficos box plot, seleciona os centros dos clusters a partir desses parâmetros e associa os intervalos a conjuntos e regras de inferência fuzzy, para converter as notas absolutas em CRA.

O método proposto apresenta especial utilidade em processos de avaliação de concursos e classificação acadêmica, pois a base de dados é composta por notas finais absolutas, obtidas a partir de procedimentos uniformes, utilizando escalas normalizadas e submetendo os candidatos e alunos aos mesmos exames, sob as mesmas restrições.

\section{REFERÊNCIAS BIBLIOGRÁFICAS}

[1] BAI, S. M., CHEN, S. M. Evaluating students' learning achievement using fuzzy membership functions and fuzzy rules. Expert Systems with Applications, 34(1), 2008a, p. 399-410.

[2] BAI, S. M., CHEN, S. M. Automatically constructing grade membership functions of fuzzy rules for students' evaluation. Expert Systems with Applications, 35(1), 2008b, p. 1408-1414.

[3] BISC. The Berkeley Initiative in Soft Computing. California: University of Berkeley. Disponível em: <http://www.cs.berkeley.edu/ zadeh/stimfl.html>. Acesso em: 10 out. 2014.

[4] BISWAS, R. An application of fuzzy sets in students' evaluation. Fuzzy Sets and System, 74(2), 1995, p. 187-194.

[5] CAMPOS, G.M. Estatística Prática para Docentes e Pós-Graduandos. Pró-Reitorias de Graduação e Pós-Graduação da USP. Disponível em:

<http://www.forp.usp.br/restauradora/gmc/gmc_livro/gmc_livro_cap14.html> Acesso em: 10 nov. 2014.

[6] CHANG, D. F., SUN, C. M. Fuzzy assessment of learning performance of junior high school students. In: Proceedings of the 1993 first national symposium on fuzzy theory and applications 1993, p. 10-15, Anais... Hsinchu, Taiwan, China.

[7] CHEN, S. M., LEE, C. H. New methods for students' evaluating using fuzzy sets. Fuzzy Sets and System, 104(2), 1999, p. 209-218. 
[8] CHENG, C. H., YANG, K. L. Using fuzzy sets in education grading system. Journal of Chinese Fuzzy Systems Association, 4(1), 1998, p. 81-89.

[9] CHIANG, T. T., LIN, C. M. Application of fuzzy theory to teaching assessment. In Proceedings of the 1994 second national conference on fuzzy theory and applications 1994, p. 92-97, Anais... Taipei, Taiwan, China.

[10] COMBS, W.E. The Combs method for rapid inference, 1997, Disponível em: $<$ http://athena.ecs.csus.edu/ hellerm/EEE222/Atricles/Combs_Fuzzy_Logic/Combs_Ra pid_Inference.htm>. Acesso em: 03 set. 2014.

[11] ECHAUZ, J. R., VACHTSEVANOS, G. J. Fuzzy grading system. IEEE Transactions on Education, 38(2), 1995, p. 158-165.

[12] FREUND, J.E., SIMON, G.A. Estatística aplicada: economia, administração e contabilidade. Trad. Alfredo Alves de Farias. 9 ed. Porto Alegre: Bookman, 2000.

[13] LAW, C. K. Using fuzzy numbers in education grading system. Fuzzy Sets and System, 83(3), 1996, p. 311-323.

[14] MA, J., ZHOU, D. Fuzzy set approach to the assessment of student-centered learning. IEEE Transactions on Education, 43(2), 2000, p. 237-241.

[15] TUKEY, J.W. Exploratory Data Analysis. (limited preliminary ed.), Reading, MA: Addison-Wesley, 1970.

[16] WANG, H. Y., CHEN, S. M. New methods for evaluating the answer scripts of students using fuzzy sets. In Proceedings of the $19^{\text {th }}$ international conference on industrial, engineering \& other applications of applied intelligent systems, 2006a, p. 442-451, Anais... Annecy, France.

[17] WANG, H. Y., CHEN, S. M. New methods for evaluating students' answer scripts using fuzzy numbers associated with degrees of confidence. In Proceedings of the 2006 IEEE international conference on fuzzy systems, 2006b, p. 5492-5497, Anais... Vancouver, B.C., Canada.

[18] WU, M. H. Research on applying fuzzy set theory and item response theory to evaluate learning performance. Master thesis, Department of Information Management, Chaoyang University of Technology, Wufeng, China, 2003.

[19] ZADEH, L.A. Fuzzy Sets, Information and Control, Vol. 8, 1965, p. 338-353. 